\title{
Paratesticular Myxoid Liposarcoma: A Case Report of Unusual Age of Presentation
}

Khaleel Al-Obaidy, Eman Abdulfatah*, Fulvio Lonardo and Rafic Beydoun

Department of Pathology, Detroit Medical Center/Wayne State University, Detroit, Michigan, USA

\begin{abstract}
Paratesticular liposarcomas are relatively common sarcomas in the paratesticular region, however, the myxoid variant; myxoid liposarcoma (MLS) is considered very rare. MLS peaks around the fourth-fifth decade. Herein, we describe a case of paratesticular MLS occurring in a 19-year-old African American male who underwent resection of a slowly growing testicular mass over a period of one and a half years that was considered initially by his primary care physician to be a benign lesion. The resection margins were positive for tumor and the patient developed local recurrence one year after the initial resection. An extremely unusual age of presentation along with the importance of margin status in tumor recurrence is highlighted.
\end{abstract}

Keywords: Myxoid Liposarcoma; Tumor; Testicular Mass

\section{Introduction}

Paratesticular sarcomas are rare and account for about $2 \%$ of all soft tissue sarcomas [1,2]. The most common type of sarcomas in this location are the liposarcomas, followed by leiomyosarcomas and rhabdomyosarcomas [2-4]. Histologically, liposarcomas are divided into four subtypes: well-differentiated, pleomorphic, myxoid/round cell and de-differentiated liposarcomas. Paratesticular myxoid/round cell liposarcomas are considered very rare, accounting for approximately $3.3 \%$ of liposarcomas in this anatomic location and is usually seen in the fourth-fifth decade $[5,6]$.

We report a case of recurrent myxoid/round cell liposarcoma in a young adult patient after surgical excision and adjuvant chemotherapy and radiation.

\section{Case Report}

A 19-year-old African American male presented with a scrotal lump that was progressively increasing in size over a period of one year and a half. There was no history of trauma, fever, or weight loss. He initially presented to his primary care physician when he discovered the mass, almost one year before he was admitted to our facility. The mass was considered a benign lesion and no further work up was done. All preoperative laboratory tests were within normal range, including complete blood count, chemistry and hormonal profile. Scrotal ultrasound showed a solid extra-testicular hypoechoic mass (Figure 1). During surgery, an $11 \times 7 \times 5 \mathrm{~cm}$ mass around an intact left spermatic cord was found and was easily removed after ligature of its blood supply.

Gross examination revealed an $11-\mathrm{cm}$ well circumscribed tumor

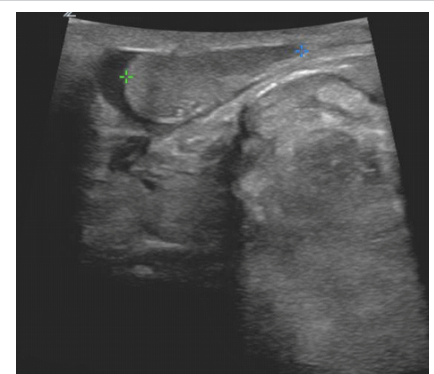

Figure 1: Scrotal ultrasonography showing solid extra-testicular hypoechoic mass.

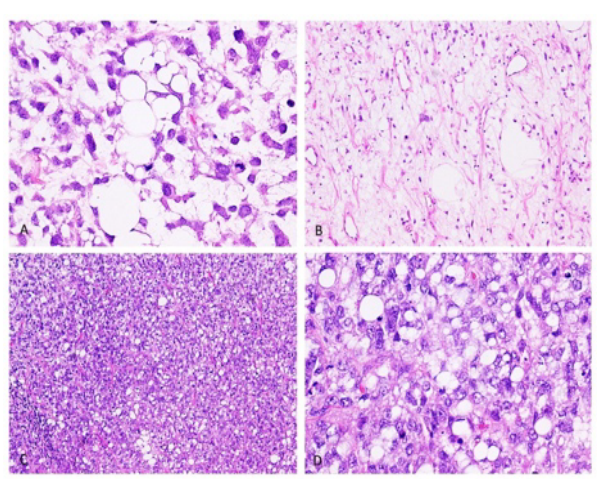

Figure 2A-D: Microscopically, the neoplastic mass shows lipoblasts with microvacuolated cytoplasm in a myxoid background and round cell morphology.

with gelatinous, gray-white nodular cut surfaces. Small areas of necrosis and hemorrhage were present. Microscopic examination showed a cellular neoplasm composed of lipoblasts in different stages of maturation with interspersed delicate thin walled arborizing and curvilinear blood vessels within a myxoid background. About 40-50\% of the tumor exhibited a round cell morphology with sheets of round to oval cells with high $\mathrm{N}: \mathrm{C}$ ratio, nuclear pleomorphism, frequent mitoses (21 mitoses/10 HPF), and minimal necrosis (Figures 2A-D). The tumor extended to resection margins. Immunohistochemical staining showed diffuse positive staining for $\mathrm{S} 100$ and vimentin and negative staining for DOG-1. Ki-67 proliferative index was about 50\%. Fluorescence in situ hybridization (FISH) using LSI DDIT3 dual color break-apart DNA probe for detection of rearrangement associated with DDIT3 gene in the $12 \mathrm{q} 13$ region was done. While FISH didn't produce a hybridization pattern, 3 intact copies of DDIT3/12q13 were detected in approximately

*Corresponding author: Eman Abdulfatah, Department of Pathology, Detroit Medical Center/Wayne State university, Detroit, Michigan, USA, Tel: +1 (313) 577 3577; E-mail: eabdulfa@med.wayne.edu

Received March 23, 2017; Accepted April 23, 2017; Published April 25, 2017

Citation: Al-Obaidy K, Abdulfatah E, Lonardo F, Beydoun R (2017) Paratesticular Myxoid Liposarcoma: A Case Report of Unusual Age of Presentation. J Mol Biomark Diagn 8: 340. doi: 10.4172/2155-9929.1000340

Copyright: (c) 2017 Al-Obaidy K, et al. This is an open-access article distributed under the terms of the Creative Commons Attribution License, which permits unrestricted use, distribution, and reproduction in any medium, provided the original author and source are credited. 


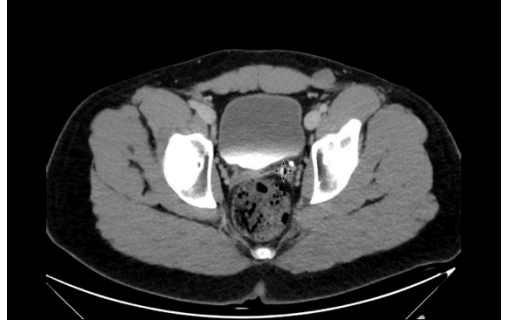

Figure 3: Computed tomography (CT) of the abdomen and the pelvis showing soft tissue nodule in the left anterolateral pelvic wall.

$39 \%$ of cells, suggesting polysomy of chromosome 12. Follow-up postoperative CT scan of thorax and abdomen showed no evidence of local disease or distant metastasis. Subsequently, left orchiectomy was performed a month later and revealed no residual disease. The patient underwent 3 cycles of adriamycin and ifosfamide adjuvant chemotherapy with radiation for the positive margins and high round cell component. One year later, CT scan of the pelvis showed a $2.3 \mathrm{~cm}$ soft tissue nodule in the left anterolateral pelvic wall, lateral to the left rectus muscle (Figure 3 ). The ultrasound guided biopsy and subsequent resection of the nodule confirmed the recurrence of myxoid/round cell liposarcoma with round cell component constituting $80 \%$ of tumor volume. Repeat FISH analysis didn't show the DDIT3 translocation, however, chromosomal analysis showed complex karyotypes including partial loss of chromosome 12. To date, the patient is back to his normal activity and been followed up in the last six months by clinical visits and CT imaging with no evidence of recurrence.

\section{Discussion}

Paratesticular sarcomas are rare and account for about $2 \%$ of all soft tissue sarcomas. The most common type of sarcomas in this location are liposarcomas (20-56\%), followed by leiomyosarcomas (19$32 \%)$ and rhabdomyosarcomas (11-24\%). Patients with paratesticular liposarcomas usually present in the fourth-fifth decade. A palpable scrotal or inguinal mass being the most common finding that are often confused with benign diseases such as hydroceles or inguinal hernias. Histologically, liposarcomas are divided into four subtypes: welldifferentiated, pleomorphic, myxoid/round cell and de-differentiated liposarcomas.

Myxoid/round cell liposarcoma is considered very rare, accounting for approximately 3.3\% [5,7]. Microscopically, it shows lipoblasts in different degree of maturation, delicate thin walled arborizing and curving blood vessels present in a myxoid background. Several prognostic factors have been reported to significantly affect the survival including: percent of round cell component, necrosis, mitosis, MIB-1 labeling index, and tumor grade [8]. Of those factors, the presence of $>5 \%$ round cell component portends a worse prognosis [9].

The diagnosis of the myxoid/round cell liposarcoma can be challenging especially in its low grade myxoid predominant form and when it is encountered in a rare location. The histologic differential diagnoses include benign and malignant lesions such as aggressive angiomyxoma, low grade myxofibrosarcoma, or low-grade fibromyxoid sarcoma.

Giving the locally aggressive nature and the infiltrative growth pattern, aggressive angiomyxoma is considered in the differential diagnosis of myxoid/round cell liposarcoma. This tumor is composed of fibromyxoid matrix sparsely populated by bland-looking spindled and stellate cells with delicate cytoplasmic processes. These features and the presence of haphazardly scattered small and large blood vessels, some of which exhibited hypertrophy or hyalinization of the wall help differentiate it from MLS [10,11].

In contrast to MLS, low-grade fibromyxoid sarcoma is composed of bland tumor cells in a variable myxoid and collagenous stroma. Typically, delicate plexiform vasculature with perivascular sclerosis, hyalinizing rosettes, strong cytoplasmic staining with MUC4 are present [12]. Finally, low grade myxofibrosarcoma, on the other hand, is characterized by fusiform tumor cells, with indistinct cell borders, slightly eosinophilic or vacuolated cytoplasm, and varying degree of hyperchromatic atypical nuclei, set in a myxoid or fibromyxoid matrix containing elongated, curvilinear capillaries with perivascular cell condensation [13-18]. However, the absence of lipoblasts and diffuse positive staining for $\mathrm{S} 100$ aid the diagnosis.

While the majority of high-grade sarcomas has complex karyotypes, most of the Myxoid/round cell liposarcomas are characterized by $t(12 ; 16)(q 13 ; q 11)$, however, the presence of polysomy of chromosome 12 has been reported in $89 \%$ of spindle/pleomorphic lipomas.

Myxoid/round cell liposarcoma has a tendency to recur locally, due to its intermediate malignant behavior. However, it rarely metastasizes to distant organs. The risk of local recurrence, sometimes after a long latency period, is similar to that of high grade lesions. Current treatment recommendations include radical orchiectomy with wide local resection of surrounding soft tissues. Adequate treatment of tumor recurrence often involves wide inguinal re-excision with complete orchiectomy if not performed previously. The importance of adjuvant radiation and chemotherapy is not well established due to the rarity of the disease.

In our case, the tumor presented at an unusual age after it was considered a benign lesion initially. Although it was negative for the characteristic translocation, our case was positive for polysomy of chromosome 12 in the initial tumor and complex karyotypes involving partial loss of chromosome 12 in the recurrent tumor. However, inadequate surgical resection margins can be postulated to be a reason behind the local recurrence of the disease one year after the initial surgical resection despite the management with adjuvant chemotherapy and radiation.

\section{References}

1. Stojadinovic A, Leung DH, Allen P, Lewis JJ, Jaques DP et al. (2002) Primary adult soft tissue sarcoma: time-dependent influence of prognostic variables. J Clin Oncol 20: 4344-4352.

2. Dotan ZA, Tal R, Golijanin D, Snyder ME, Antonescu C, et al. (2006) Adult genitourinary sarcoma: the 25-year Memorial Sloan-Kettering experience. J Urol 176: 2033-2038.

3. Khoubehi B, Mishra V, Ali M, Motiwala H, Karim O (2002) Adult paratesticular tumours. BJU Int 90: 707-715.

4. Mondaini N, Palli D, Saieva C, Nesi G, Franchi A, et al. (2005) Clinica characteristics and overall survival in genitourinary sarcomas treated with curative intent: a multicenter study. Eur Urol 47: 468-473.

5. Montgomery E, and Fisher C (2003) Paratesticular liposarcoma: a clinicopathologic study. Am J Surg Pathol. 27: 40-47.

6. Fletcher CDM, Bridge JA, Hogendoorn P, Mertens F (2013) WHO Classification of Tumours of Soft Tissue and Bone IARCPress, France, UK.

7. Fitzgerald S, and MacLennan GT (2009) Paratesticular liposacroma. J Urol 181: 331-332.

8. Tateishi U, Hasegawa T, Beppu Y, Kawai A, and Moriyama N (2003) Prognostic significance of grading (MIB-1 system) in patients with myxoid liposarcoma. Journal of Clinical Pathology. 56: 579-582.

9. Smith TA, Easley KA, and Goldblum JR (1996) Myxoid/round cell liposarcoma 
Citation: Al-Obaidy K, Abdulfatah E, Lonardo F, Beydoun R (2017) Paratesticular Myxoid Liposarcoma: A Case Report of Unusual Age of Presentation. J Mol Biomark Diagn 8: 340. doi: 10.4172/2155-9929.1000340

of the extremities. A clinicopathologic study of 29 cases with particular attention to extent of round cell liposarcoma. Am J Surg Pathol 20: 171-180..

10. Quesada J, and Amato R (2012) The Molecular Biology of Soft-Tissue Sarcomas and Current Trends in Therapy. Sarcoma 2012:1-16.

11. Tsang WY, Chan JK, Lee KC, Fisher C, Fletcher CD (1992) Aggressive angiomyxoma. A report of four cases occurring in men. Am J Surg Pathol 16: 1059-1065.

12. Folpe AL, Lane KL, Paull G, Weiss SW (2000) Low-grade fibromyxoid sarcoma and hyalinizing spindle cell tumor with giant rosettes: a clinicopathologic stud of 73 cases supporting their identity and assessing the impact of high-grade areas. Am J Surg Pathol 24: 1353-1360.

13. Huang HY, Lal P, Qin J, Brennan MF, Antonescu CR (2004) Low-grade myxofibrosarcoma: a clinicopathologic analysis of 49 cases treated at a single institution with simultaneous assessment of the efficacy of 3-tier and 4-tier grading systems. Hum Pathol 35: 612-621.
14. Mentzel T, Calonje E, Wadden C, Camplejohn RS, Beham A, et al. (1996) Myxofibrosarcoma. Clinicopathologic analysis of 75 cases with emphasis on the low-grade variant. Am J Surg Pathol 20: 391-405.

15. Weaver J, Downs-Kelly E, Goldblum JR, Turner S, Kulkarni S, et al. (2008) Fluorescence in situ hybridization for MDM2 gene amplification as a diagnostic tool in lipomatous neoplasms. Mod Pathol 21: 943-949.

16. Cotran RS, Kumarand V, Collins T (1999) Robbins Pathologic Basis of Disease (6 edn), W. B. Saunders Co, Philadelphia, USA.

17. Chung PW, Deheshi BM, Ferguson PC, Wunder JS, Griffin AM, et al. (2009) Radiosensitivity translates into excellent local control in extremity myxoid liposarcoma: a comparison with other soft tissue sarcomas. Cancer 115: 3254-3261.

18. Nassif NA, Tseng W, Borges C, Chen P, Eisenberg B (2016) Recent advances in the management of liposarcoma. F1000Research 5: 2907. 